\title{
Possibility of Total lonizing Dose Effects measurements for LHC experiments elements in a medical facility: the TIFPA-INFN experience
}

\author{
Benedetto Di Ruzza ${ }^{a, *}$ \\ ${ }^{a}$ Trento Institute for Fundamental Physics and Applications (TIFPA-INFN), \\ Via Sommarive 14, 38123 Trento, Italy \\ E-mail: benedetto.diruzza@tifpa.infn.it \\ ORCID:0000-0001-9925-5254
}

Total Ionizing Dose (TID) Effects tests are mandatory requirements not only for solid state particle sensors development but also for electronic elements and control systems progress in all the LHC detectors. Both present and future upgraded elements have to satisfy predefined validation procedure generally performed in irradiation facilities. Now-days these tests and studies can be performed not only in facilities explicitly built for this mission, but with some wisdom, also in medical or biological research facilities when some minima requirements are satisfied. In this article will be shown the planification and realization of SiPM X-ray irradiations for TID studies and characterization realized in the Italian TIFPA-INFN Trento Center laboratory, using instruments originally realized for medical or biological irradiations. In detail will be described the minimum flexibility required by the x-ray irradiation set-up and by the dose measurement system in order to perform this double task and finally the irradiation planification and realization.

The Ninth Annual Conference on Large Hadron Collider Physics - LHCP2021

7-12 June 2021

Online

"Speaker 


\section{Introduction}

The Trento Institute for Fundamental Physics and Applications (TIFPA)[1] is a multidisciplinary Research Center located in Trento (Italy) and operated by the Italian National Institute for Nuclear Physics (INFN). One of the laboratories of the center is the x-ray irradiation laboratory (realized in 2015) equipped with a $3 \mathrm{~kW}$ irradiation station and a absolute dose measurement system based on a calibrated farmer chamber. The irradiation system is based on a 30-195 kV x-ray tube with tungsten (W) anode. The irradiation laboratory was realized for biological and biophysics cellular irradiation studies, and is commonly used by TIFPA and CIBIO[2] researchers. For biological and biophysics irradiations are often used tungsten $\mathrm{K}$ emission lines, that's why the TIFPA $\mathrm{x}$-ray tube is generally used at $195 \mathrm{kV}, 10 \mathrm{~mA}$ current with a $3 \mathrm{~mm} \mathrm{Al}$ or $1 \mathrm{~mm} \mathrm{Cu}$ radiation filter and in each irradiation session, generally a dose of 5-20 Gy is delivered on the cells samples. In November 2019 begun the investigation about the possibility of using this laboratory also for non-biological irradiation, and after some preliminary requirements verification, arose the possibility of use almost the same set-up (with only few changes) for Total Ionizing Dose (TID) effects studies and characterization on silicon sensors and electronic devices. This article will summarize the tests performed in order to achieve this goal and will describe the final configuration used in May 2021 to perform a TID characterization on innovative Silicon Photo-multiplier (SiPM) designed and fabricated by the Bruno Kessler Foundation (FBK) Research Institute [3] located in Trento.

\section{X-rays Irradiation Station mandatory requirements for TID studies}

In order to perform TID dose studies on silicon sensors, particle tracking systems and electronic devices using x-rays emitted by a tungsten anode, due to the thickness of the sensors, tungsten $\mathrm{L}$ emission lines have to be used. To optimize the $\mathrm{x}$-ray tube emission intensity in this spectrum range, the maximum tube voltage and the spectrum filter thickness have to be carefully selected. This brings to the two mandatory requirements in order to modify existent x-ray irradiation for TID studies on sensors: 1) the possibility to set the maximum tube voltage no greater than $40 \mathrm{kV} ; 2$ ) the possibility to change the existing installed radiation filter out of the tube window exit. In the TIFPA laboratory this optimization was performed using the SpekPy[4] simulation software. The final values of these two parameters selected for the TIFPA $\mathrm{x}$-ray tube were $40 \mathrm{kV}$ maximum voltage and $0.180 \mathrm{~mm}$ thickness Al filter [see Fig.(1) left].

\section{Delivered dose measurement system}

In irradiation studies for silicon sensors and electronic devices the de facto standard dose measurement system is the one used at CERN [5] based on the measurement of the current created by the radiations in calibrated silicon diodes located in the irradiation spot. In order to compare the results between different laboratories, is suggested to duplicate the same system in all the other irradiation facilities. Due to lack of time for installing this new diode based dose measurement system, in the TIFPA laboratory, was decided to use the already existing dose measurement system, based on farmer chamber, that is the standard for absolute dose measurement in biological and biophysics irradiations. The equivalence of the two dose 
measurement system was tested in the X-rays Irradiation Laboratory located in the Department of Physics and Astronomy 'Galileo Galilei' of the Padova University (Italy). This laboratory, used only for TID studies, is equipped with a calibrated CERN diode dose measurement system and a tungsten irradiation tube where was possible to set the same tube max voltage, filter and current required for the TIFPA TID irradiations. In March 2021, in the Padova x-rays irradiation station were performed multiple irradiations using together the TIFPA farmer chamber and the CERN diodes together [see Fig.(1) right] putting both of them in the center of the spot and at the same distance from the tube planed for the TIFPA irradiations. Keeping fixed all the other tube parameters and changing only the tube current, the measurements showed a fixed ratio $\mathrm{R}=($ farmer chamber dose)/(calibrated silicon diode dose) for tube current between 10-30mA. This $\mathrm{R}$ value was then used to convert the farmer chamber dose read-out to calibrated diode dose value in the irradiations that toke place in the TIFPA laboratory on May 2021.
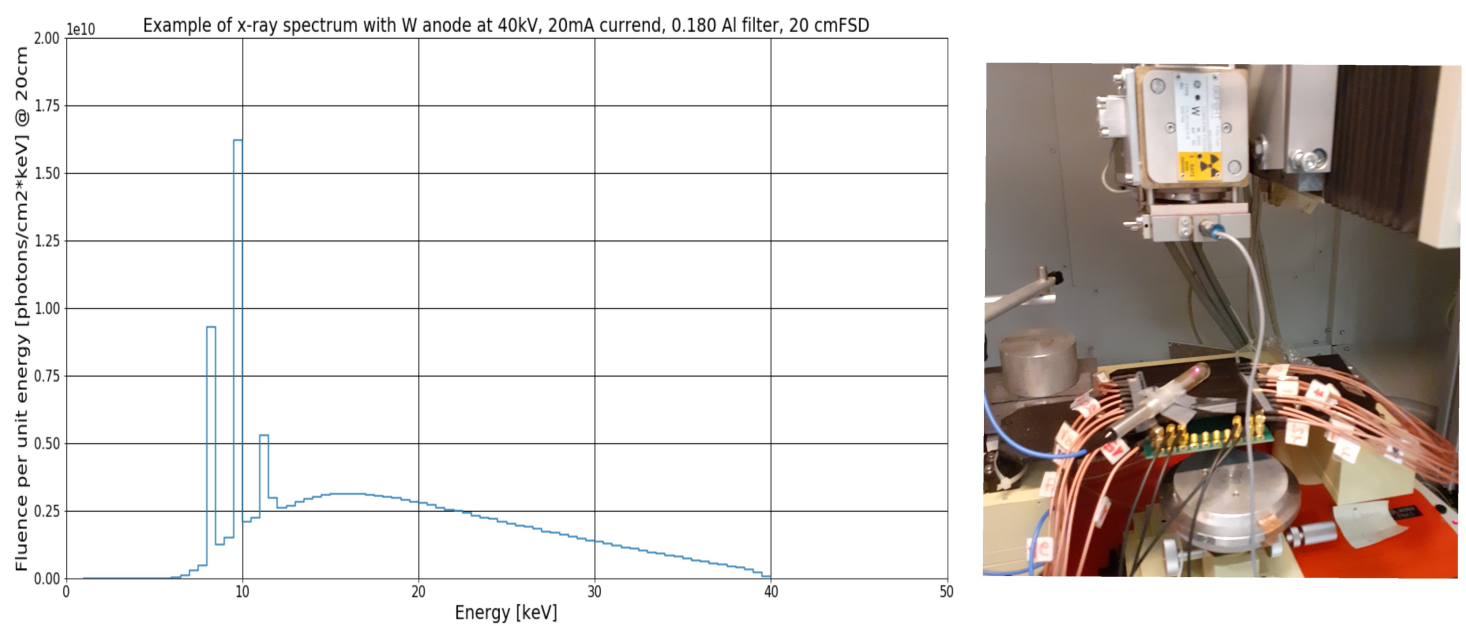

Fig.(1): X-ray spectrum simulation (left) and cross measurement dose set-up (right) used in the Padova X-rays Irradiation Laboratory.

\section{SiPM irradiation set-up}

After these investigation, in April 2021, a successful irradiation and characterization campaign started on a batch of brand new FBK designed and produced SiPM. The TIFPA x-ray tube was equipped with a new $0.180 \mathrm{~mm} \mathrm{Al}$ filter and the final DUTs supports for the SiPM were realized using a 3D printer. A specific FBK characterization equipment for SiPM, placed close to the irradiation cabinet, was connected to the DUTs located in the irradiation volume using a cables serpentine path already present in the irradiation station shielding material (used for the dose measurement probe connection). The SiPM characterization set-up and the real-time dose measurement system was controlled remotely using a ethernet connection during the irradiation. Using this set-up was possible not only to perform characterization between the different irradiation sessions (without moving the DUTs) but also preliminary annealing measurements.

Using this set-up, represented in Fig.(2), 10Mrad(Si) dose were delivered in three working days on the DUTs. 

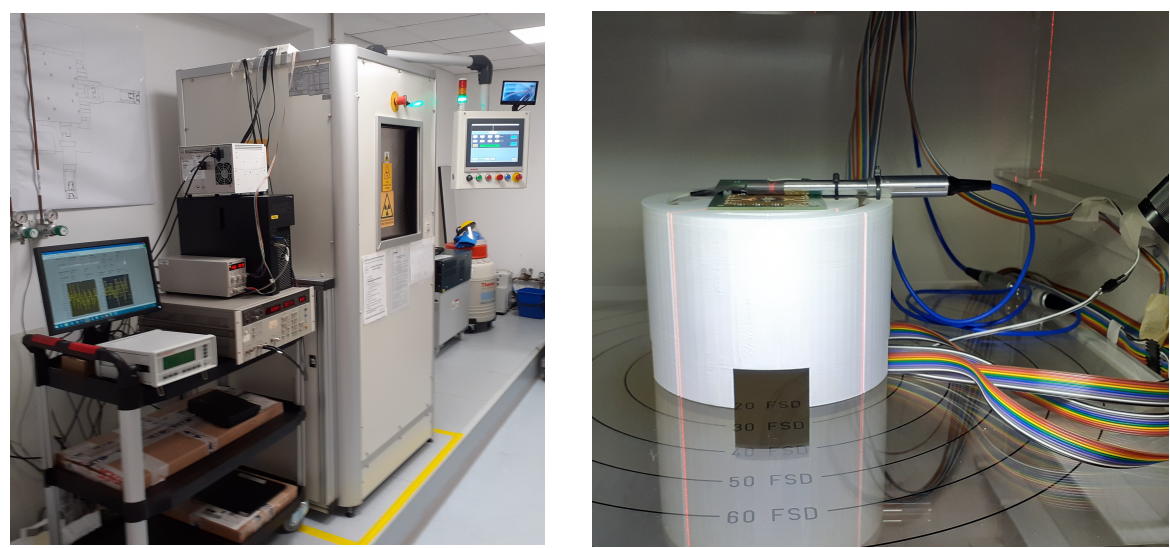

Fig.(2): Irradiation and monitoring set-up (left) and DUT detail (right).

\section{Conclusions}

A medical or biological $\mathrm{x}$-ray irradiation facility can be easily converted in an irradiation facility for TID studies if the x-ray tube satisfies at least the following two conditions: 1) the possibility to set the maximum tube voltage no greater than $40 \mathrm{kV} ; 2$ ) the possibility to change the existing installed radiation filter out of the tube window.

Applying carefully the preliminary discussed comparison, a calibrated farmer chamber can be used as delivered dose measurement system in a TID irradiation session if a calibrated diode dose system is temporary not available.

Many TID studies are required in developing the elements of future experiments upgrades at LHC. The possibility of using also medical and biological facilities for these studies, in addition to the facilities already explicitly planned for this goal, can speed-up consistently the realization of the projects, especially in the R\&D stages of the new detectors construction.

\section{Acknowledgments}

The author thanks Federico Faccio (CERN), Emanuele Scifoni (TIFPA-INFN) and Walter Tinganelli (GSI) for the suggestions and the useful discussions. This work was realized with the crucial help of Serena Mattiazzo, Devis Pantano and Jeff Wyss (INFN-PD); Anna Rita Altamura, Fabio Acerbi and Alberto Mazzi (FBK Trento). Thanks also to Giuseppe Battistoni (TIFPA-INFN) and Rita Dolesi (University of Trento) for the financial support to this project.

\section{References}

[1] Trento Institute for Fundamental Physics and Applications (TIFPA): www . tifpa.infn. it

[2] Cellular, Computational and Integrative Biology Research Center (CIBIO), University of Trento: https://www.cibio.unitn.it

[3] Bruno Kessler Foundation (FBK): https://www. fbk.eu/en

[4] G Poludniowski, A Omar, R Bujila and P Andreo, Technical Note: SpekPy v2.0-a software toolkit for modeling $x$-ray tube spectra. Med Phys. 2021; https://doi.org/10.1002/mp.14945

[5] CERN EP-ESE irradiation system:

https://espace.cern.ch/project-xrayese/_layouts/15/start.aspx\# 